\title{
AVALIAÇÃO DA SOBRECARGA DOS CUIDADORES INFORMAIS ATRAVÉS DA ESCALA DE BURDEN INTERVIEW ATENDIDOS DURANTE O ESTÁGIO DE FISIOTERAPIA EM SAÚDE COLETIVA
}

\author{
EVALUATION OF THE OVERLOAD OF INFORMAL CAREGIVERS THROUGH THE BURDEN IN- \\ TERVIEW SCALE ATTENDED DURING THE PHYSIOTHERAPY STAGE IN COLLECTIVE HEALTH
}

\author{
Adriano Rodrigues Oliveira $a^{a^{*}}$, Giuliano Roberto Gongalvez ${ }^{\mathbf{b}^{* *}}$, \\ Maria de Castro Monteiro Loffredo ${ }^{c^{*}}$, Leandro Henrique Grecco ${ }^{\mathrm{d}^{* * *}}$ \\ prof.adrianoro@gmail.coma, giulianoanato@gmail.com ${ }^{\mathrm{b}}$ marialoffredo78@hotmail.com ${ }^{\mathrm{c}}$, leandrohgrecco@gmail.com ${ }^{\mathrm{d}}$ \\ Universidade Nove de Julho*, Centro Universitário UniMetrocamp**, Faculdade São Leopoldo Mandic ${ }^{* * *}$
}

\section{RESUMO}

Introdução e Objetivo: O objetivo do estudo foi identificar a sobrecarga imposta ao cuidador informal através da escala de Burden Interview, de pacientes atendidos durante o estágio de fisioterapia em saúde coletiva. Materiais e Métodos: Foram selecionados 15 cuidadores de pacientes com dependência total ou dependência modificada, de acordo com a escala de MIF, os quais responderam ao questionário Burden Interview. Resultados: Analisando os resultados, observa-se uma sobrecarga imposta a esses cuidadores em todos os domínios da escala (tensão geral, isolamento, decepção, envolvimento emocional e ambiente), bem como no score total 47,27 $( \pm 14,94)$. Desta forma, concluímos que o cuidador sofre uma sobrecarga física e emocional, apontando que apesar de contribuirmos para uma melhor qualidade de vida do internado, pode-se criar uma situação desfavorável ao cuidador. Conclusões:Por esse motivo, acreditamos que nosso estudo pode ser uma ferramenta válida para corroborar com a importância do atendimento multidisciplinar e programas de saúde específicos ao cuidador informal e não apenas ao ser cuidado.

Palavra chave: Idoso, burden interview, cuidadores, assistência domiciliar, internação domiciliar.

\begin{abstract}
Introduction and Objectives:The aim of the study was to identify the overload imposed on the informal caregiver through the Burden Interview scale of patients attended during the physiotherapy stage in collective health. Materiais e Métodos: Fifteen caregivers of patients with total dependence or modified dependence were selected according to the MIF scale, who answered the Burden Interview questionnaire. Results: Analyzing the results, it is observed an overload imposed to these caregivers in all domains of the scale (general tension, isolation, disappointment, emotional involvement and environment), as well as in the total score 47,27 $( \pm 14,94)$. In this way, we conclude that the caregiver suffers a physical and emotional overload, pointing out that although we contribute to a better quality of life of the hospitalized, it can create a situation unfavorable to the caregiver. Conclusions:For this reason, we believe that our study may be a valid tool to corroborate with the importance of multidisciplinary care and health programs specific to the informal caregiver and not only when being cared for.
\end{abstract}

Key words: Elderly, burden interview, caregivers, home care, home hospitalization. 


\section{INTRODUÇÃO}

Segundo dados do IBGE, nunca nosso mundo atingiu um numero tão grande de idosos, até o meio do século XXI estima-se que em países desenvolvidos a expectativa de vida seja para homens 87,5 anos e para mulheres 92,5 anos e em países em desenvolvimento 82 anos para homens e 86 para as mulheres, com essas informações vimos que a proporção de idosos está aumentando em todos os países. Isto acontece devido à diminuição da fecundidade, a medicina preventiva e os programas voltados à qualidade de vida. $\mathrm{O}$ Brasil está em um patamar importante, estima-se que até 2025 alcançará o sexto lugar com o maior numero de pessoas idosas no mundo, daí a importância para conhecermos o processo de envelhecimento da nossa população. (1)

Assim, o envelhecimento que antes era considerado um fenômeno, hoje faz parte da maioria da nossa sociedade, podendo ser compreendido como um processo natural da vida, onde acontece um estresse do organismo devido a estímulos intrínsecos e extrínsecos, tornando-o menos eficiente funcionalmente e em sua restauração, deixando-o mais frágil para enfrentar o meio ambiente, favorecendo o aumento de doenças crônicas e degenerativas. (2-4)

As complicações decorrentes do envelhecimento podem acarretar em condições de doenças como Acidente Vascular Encefálico (AVE), Demência, Mal de Parkinson ou Alzheimer, propiciando uma condição que requeira assistência médica e por sua vez internação, seja hospitalar ou domiciliar.(4)

A doença, independente de qual seja, é sempre um acontecimento estressor que produz efeitos no doente e nos familiares, que ao vivenciar a crise provocada pela doença, experimenta inicialmente um desequilíbrio em sua capacidade de funcionamento normal, provocando alterações que envolvem afeto, finanças e social, levando uma reorganização familiar. $(5,6)$

Os custos sociais e financeiros para os idosos e seus familiares frente a essas doenças são elevados, pois quando se comparado ao jovem, os idosos tem um tempo de internação maior, a reabilitação é mais complicada e extensa, correndo o risco de ser institucionalizados e, desta forma, perdendo sua autonomia, sua independência e posteriormente gerando o risco de morte. Esses fatores associados levam a quadros de baixa autoestima e depressão, diminuindo consideravelmente a qualidade de vida do idoso (7).

Com já dito, apesar da doença afetar toda a família, normalmente a responsabilidade principal sobre o cuidado do paciente recai sobre um único familiar, denominado cuidador principal. Ele tem que lidar não só com as dificuldades do paciente com a mobilidade, autocuidado e comunicação, como também com os possíveis déficits cognitivos, depressão e mudança na personalidade. (8)

Desta forma, os pacientes submetidos ao regime domiciliar são assistidos pelo cuidador, cujo termo se aplica à realidade de pessoas que prestam cuidados em prevenção, proteção e recuperação da saúde, de maneira formal ou informal. (9)

Didaticamente, temos dois tipos de cuidadores, o formal e o informal. O cuidador formal é aquele que possui conhecimentos adquiridos em treinamentos direcionados, referentes à profissão, em geral recebendo remuneração em troca de seus serviços seja ele no hospital, clínica ou home care. O cuidador informal é representado pelo segmento leigo, normalmente um membro da família, o que resulta na expressão familiar cuidador. Essa pessoa assume a responsabilidade, dedicando grande parte de seu cotidiano a rotinas de cuidado com a pessoa doente, com pouco ou quase nenhum conhecimento técnico, mas em resposta às necessidades que as condições de vida lhe impõem. (9)

No entanto, este cuidador informal absorve uma sobrecarga, a qual é caracterizada pelo fato de o cuidador familiar se ver sem apoio e permanentemente diante de situações de enfrentamento, ocasionando queixas de ordem objetiva e subjetiva, associada ao caso do cuidado realizado. (10)

A sobrecarga objetiva refere-se às consequências negativas geradas pelo papel do cuidador, como alterações na rotina, diminuição da vida social e profissional. Já a sobrecarga subjetiva remete as percepções, preocupações, sentimento negativo e incômodo gerado por tornar-se um cuidador de um paciente dependente. $(10,11)$

Existem várias maneiras de se avaliar as sobrecargas impostas, como por exemplo, fazendo uso das escalas Self-Questionare-20 (SRQ-20), Per- 
turbação do Comportamento e Humor (BMD), Escala de Sobrecarga de Familiares de Pacientes Psiquiátricos (FBIS-BR) e a escala de Burden Interview (BI). (6,10-12)

Dentre essas escalas, destacamos a escala de BI, que teve a sua tradução e validação para a língua portuguesa e trata-se de uma escala objetiva, composta por 22 questões, sendo a mais utilizada para a avaliação de sobrecarga em cuidadores de pacientes idosos. Apesar de ser desenvolvida a partir da experiência de seus autores em clínicas e pesquisas com pacientes idosos com demência e seus familiares, seus itens são abrangentes e tratam de dimensões comuns e de várias doenças físicas e mentais. (10-12)

A fisioterapia em domicílio consiste em proporcionar ao paciente prevenção e promoção da saúde, bem como a reabilitação de seu quadro clínico. Entretanto, a assistência por vezes limita-se apenas ao ser cuidado, favorecendo a uma possível sobrecarga física e emocional ao cuidador e por esse motivo, acreditamos que nosso estudo possa identificar o perfil desses cuidadores e a sobrecarga que lhe é imposta, possibilitando dessa forma novas estratégias e visão sob o regime de atendimento domiciliar.

$\mathrm{O}$ objetivo geralIdentificar a sobrecarga dos cuidadores informais através da através da Burden Interview, responsáveis pelos cuidados diários de pacientes internados em regime domiciliar, os quais são atendidos durante o estágio de fisioterapia em saúde coletiva.

Já o objetivo específico buscou caracterizar demograficamente a população que compõe os cuidadores informais dos pacientes que são atendidos durante o estágio de fisioterapia em saúde coletiva.

\section{MATERIAIS E MÉTODO}

\section{TIPO DE ESTUDO/ LOCAL}

Trata-se de um estudo transversal de abordagem qualitativa, administrado na residência dos pacientes que estão sob regime de internação domiciliar, cadastrados no estágio de fisioterapia em saúde coletiva da Universidade Nove de Julho (UNINOVE), em São Paulo, SP.

\section{AMOSTRA}

Para definição do número de sujeitos entrevistados utilizou-se do critério de saturação ou recorrência dos dados, através da lista de espera em saúde coletiva para atendimento domiciliar da UNINOVE. Os selecionados responderam ao questionário avaliativo com tempo estimado de resposta de 30 minutos.

\section{CRITÉRIOS DE INCLUSÃO}

- Ser um cuidador informal de pacientes crônicos sob-regime de internação domiciliar;

- O paciente tem que apresentar encaminhamento médico para fisioterapia;

- O paciente deve ter duração mínima de incapacidade de 4 meses, bem como o cuidador apresentar o mesmo período de assistência;

- Apresentar interpretação de score da Medida de Independência Funcional (MIF) como dependência completa, com necessidade de assistência total ou dependência modificada, com assistência de até $50 \%$ nas tarefas funcionais;

- Estar de acordo e assinar o Termo de Consentimento Livre Esclarecido (TCLE).

\section{CRITÉRIOS DE EXCLUSÃO}

- Se o cuidador apresentar complicações ortopédicas, cardiorrespiratórias ou psíquicas que impedem a execução das suas funções para com o paciente;

- Se o paciente apresentar condições clínicas que não caracterizem dependência em suas atividades de vida diária;

- Se o paciente estiver realizando qualquer outra forma de tratamento que não seja a fisioterapia domiciliar.

Se o cuidador estiver realizando outro acompanhamento que não seja da equipe de fisioterapia que forneça atendimento ao doente crônico.

Os indivíduos selecionados foram informados da possibilidade de serem retirados da pesquisa em qualquer fase da mesma, sem penalização alguma, antes mesmo de lerem e assinarem o Termo de Consentimento (Anexo I) para participação da pesquisa clínica, conforme aprovado pelo Comitê de Ética em Pesquisa (segundo a resolução CNS 196/96, do Conselho Nacional de Saúde, de 10/10/96, protocolo 18402 datado em $07 / 05 / 2012$ ).

\section{PROCEDIMENTO/ PROTOCOLO}

O trabalho foi realizado com triagem dos indivíduos, conforme critérios de inclusão. Ao fi- 
nalizar a parte informativa (Termo de Consentimento), foi ministrada uma orientação sobre as atividades a serem executadas durante a coleta dos dados, bem como a finalidade do trabalho.

\section{AVALIAÇÃO COM QUESTIONÁRIOS}

\section{Burden Interview}

A escala de Burden Interview é composta por 22 perguntas, divididas em cinco conceitos (ou dimensões) de saúde: Tensão Geral (8 itens), Isolamento (3 itens), Decepção (5 itens), Envolvimento Emocional (3 itens) e Ambiente (3 itens). Cada componente deverá ser respondido com as seguintes opções: 1- de modo algum; 2- raramente; 3- algumas vezes; 4- frequentemente. Para interpretação do score, podemos analisar cada conceito separadamente, realizando a média aritmética dos valores equivalentes às respostas das questões específicas ou analisar os conceitos totalizados, realizando a média aritmética dos valores equivalentes as respostas das 22 questões. (12) Para respondê-lo, haverá um tempo médio de aproximadamente 30 minutos e será considerado como sobrecarga do cuidador quando o valor do score for maior que 50\% do score máximo de cada componente avaliado ou no valor total da soma de todos os domínios.

\section{Medida de Independência Funcional (MIF)}

Trata-se de um instrumento amplamente utilizado internacionalmente para avaliação da capacidade funcional e tem como diferencial a incorporação da avaliação cognitiva. A escala é composta por 18 categorias agrupadas em seis dimensões: autocuidado, controle de esfíncteres, transferências, locomoção, comunicação e cognição social. Cada item tem uma pontuação de 1 a 7 , em que 1 corresponde à dependência total e 7 , à independência completa. Cada dimensão é analisada pela soma dos itens que a compõem. Nesse instrumento são descritos dois domínios: o motor, referente às dimensões de autocuidado, controle de esfíncteres, transferências e locomoção; e o cognitivo, que engloba as dimensões de comunicação e cognição social. (13)

O escore total da MIF é obtido somando-se a pontuação de cada dimensão, sendo que o mínimo é de 18 e o máximo, de 126 pontos. Até 18 pontos, considera-se que há dependência completa, com necessidade de assistência total; de 19 a 60 pontos, dependência modificada, com assistência de até $50 \%$ na tarefa; de 61 a 103 pontos, dependência modificada, com necessidade de assistência de até $25 \%$ na tarefa e de 104 a 126 pontos, equivalente a independência completa/modificada. Para respondê-lo, o cuidador e/ou paciente terá um tempo aproximado de 30 minutos.(14)

\section{PROCEDIMENTOS APÓS A REALIZAÇÃO DA PESQUISA}

Após o término da pesquisa não haveria necessidade de continuidade de atendimento aos voluntários. Entretanto, como houve interesse de ambas as partes, continuamos com os atendimentos em caráter de aprendizado universitário, agregado ao estágio em saúde coletiva.

Os dados das coletas foram arquivados em bancos de dados em arquivos do Ambulatório de Fisioterapia da UNINOVE.

\section{ANÁLISE ESTATÍSTICA}

Para realizar a interpretação do score da escala de Burden Interview, utilizamos de uma análise estatística simples, compreendendo média, desvio padrão e porcentagem, fazendo uso do programa Excel (Microsoft Office 2010) para realizar tal feito.

\section{RESULTADOS}

A amostra inicial foi constituída de 23 voluntários, dos quais 08 foram excluídos por não apresentarem a escala de MIF como dependência completa ou modifica que represente dependência em até $50 \%$ nas atividades cotidianas. Desta forma, foram selecionados 15 voluntários para realização do trabalho, sendo caracterizados na Tabela 1. 
Tabela 01: Características demográficas dos voluntários cuidadores.

\begin{tabular}{cc} 
Denominação & Valores / Características \\
Gênero & $86,66 \%$ Feminino \\
Estado civil & $46,66 \%$ Casadas \\
Idade (média/desvio padrão) & $52,53( \pm 10,48)$ \\
Escolaridade & $46,66 \%$ Ensino Médio \\
Curso de Cuidador & $93,33 \%$ - Não \\
Cuidados diários (média horas/dia) & $21,93-$ Horas por dia \\
Parentesco & $73,33 \%$ - Filhos \\
Tempo exerce cuidado (média/meses) & $8,33 \%$ - Meses \\
Divide tarifas & $53,33 \%$ - Não \\
\hline
\end{tabular}

Após a tabulação dos dados demográficos dos voluntários, os resultados referentes à aplicação do questionário MIF e perfil dos pacientes submetidos a cuidados domiciliares foram expostos na Tabela 02.

Tabela 02: Características demográficas dos pacientes submetidos aos cuidados domiciliares

\begin{tabular}{cc} 
Denominação & Valores / Características \\
Gênero & $86,66 \%$ - Feminino \\
Idade (média/desvio padrão) & $52,6( \pm 12,27)$ \\
Diagnóstico & $73,33 \% \mathrm{w}-\mathrm{AVE}$ \\
Tempo de lesão meses (média/dp) & $36( \pm 24,05)$ \\
MIF (média score/ desvio padrão) & $56,73( \pm 16,86)$ \\
\hline
\end{tabular}
$\mathrm{dp}=$ desvio padrão

$\mathrm{Na}$ Tabela 03 foram expostos os resul- Interview Scale, apontando os valores de tados referentes à aplicação da Burden sobrecarga.

Tabela 03: Valores expressos em média e desvios padrão, obtidos através da Burden Interview Scale

\begin{tabular}{ccc} 
Aspectos & Score Cuidadores & Score Máx. / Corte \\
Tensão Geral & $23,66( \pm 7,35)$ & $32 / 16$ \\
Isolamento & $8,33( \pm 2,98)$ & $12 / 06$ \\
Decepção & $12,53( \pm 3,55)$ & $20 / 10$ \\
Envolvimento Emocional & $5,26( \pm 2,69)$ & $12 / 06$ \\
Ambiente & $6,93( \pm 3,24)$ & $12 / 06$ \\
Score Total & $47,27( \pm 14,94)$ & $88 / 44$ \\
\hline
\end{tabular}

O Gráfico 1 exemplifica a tabela 3, mostrando que o perfil dos cuidadores dos pacientes dependentes influi diretamente em sobrecarga desta população. 
Gráfico 1: Gráfico representando os domínios do Burden Interview Scale, expressos em média e desvios padrão

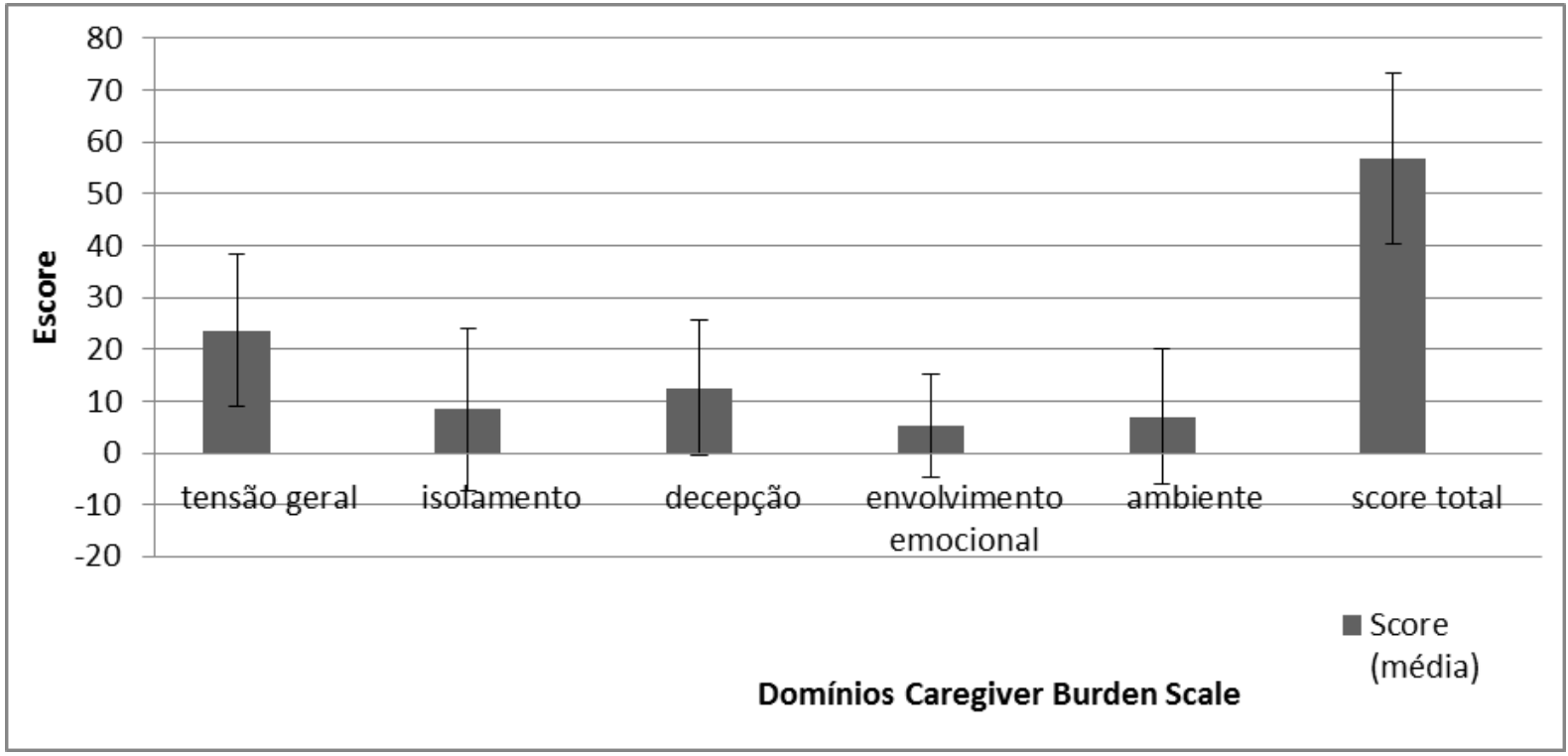

\section{DISCUSSÃO}

O papel de cuidador é construído no relacionamento, com a influência de diversos fatores referentes a historia da família. Isso indica que de alguma forma, esse papel já estava imaginado ou predeterminado. Ademais, existe uma tendência de os padrões familiares se repetirem em virtude das transmissões geracionais. Não só as expectativas, mas também os conflitos são transmitidos multigeracionalmente. (15)

Os cuidadores se deparam com numerosos fatores, que incluem a aceitação do diagnostico, lidar com um stress cada vez maior, administrar o conflito dentro da família e planejar o futuro. (16)

Diversos são os desafios que se apresentam no cotidiano do cuidador, tais como a dificuldade para lidar com os quadros de agitação e de agressividade do ser cuidado,(17) o qual pode levar a um quadro de envolvimento emocional, onde no nosso estudo há uma inclinação para sobrecarga nesse domínio $(5,26 \pm 2,69)$, pois abordam-se questões relacionadas a influencia do comportamento do paciente na vida do cuidador, como por exemplo se devido a esse comportamento o cuidador tem vergonha ou sente-se ofendido por determinadas atitudes do paciente.

A situação de doença prolongada de um familiar, muitas vezes, representa uma situação de crise geradora de stress, uma ameaça ao equilíbrio do normal funcionamento pessoal, familiar e social. (18) Tal afirmação corrobora com os resultados da nossa pesquisa, pois o cuidador desenvolve uma tensão geral acentuada $(23,66 \pm 7,35)$, tendo em vista que esses cuidadores são parentes de primeiro grau dos pacientes (73,33\% são filhos), não apresentam um curso específico para cuidar dessa população $(93,33 \%)$ e dedicam exclusividade para cuidados diários (média de 21,93 h/dia). A sobrecarga do cuidador - expressão traduzida da língua inglesa e conhecida internacionalmente como Burden Interview -, similar ao estresse, é suposta como uma reação negativa aguda à prestação de cuidados, que surge quando novas demandas de cuidados são introduzidas ou quando demandas de cuidados já existentes se intensificam. Cuidadores incapazes de se adaptar ou modificar as suas estratégias para atender as demandas de cuidados, evoluem para quadros de sobrecarga, ficando vulnerável a doença física, depressão, perda de peso e insônia (16).Tais sobrecargas são constatadas em nossa pesquisa pelo score total da escala $(47,27$ $\pm 14,94)$.

O estresse relacionado aos cuidados é um fenômeno multidimensional que engloba quatro grandes domínios: o quadro de cuidado (incluindo as características do cuidador, o tipo de relacionamento entre o cuidador e aquele que é cuidado e 
a disponibilidade de apoio social); as situações de estresse relacionadas ao cuidado, que podem ser primárias (como o comportamento problemático do paciente) ou secundárias (tal como outros compromissos do cuidador); fatores que podem servir de moderadores da percepção de estresse, como estratégias de enfrentamento e de gestão de cuidados; e as consequências dos cuidados para o bem estar geral do cuidador (19),. A escala utilizada em nosso estudo contrasta com os apontamentos dos autores, sendo que em todos os domínios abordados (tensão geral, isolamento, decepção, envolvimento emocional e ambiente) houve inclinação para a sobrecarga dos cuidadores.

As alterações fisiológicas e biológicas no idoso exigem participação e ajuda dos familiares e ou cuidadores, através da proximidade física e dos vínculos emocionais, o sistema emocional da família é profundamente abalado, passam a se impor privações e modificações no estilo de vida para incluir as novas necessidades de seu membro doente. A família se vê envolvida por sentimentos intensos e conflitantes, difíceis de manejar que acabaram por lhe impor certo isolamento (16) fato constatado em nossa pesquisa, apresentando um score de $8,33( \pm 2,98)$ para o domínio isolamento, que abrange questões como evitar o contanto com amigos e adiar planos para determinada etapa de vida devido aos problemas de saúde do paciente que se encontra sob seus cuidados.

O diagnóstico de uma patologia que afeta o sistema neurológico traz uma realidade contundente que implica em muitas perdas envolvendo a autonomia do corpo e o afastamento do eu para o individuo. Com isso, o cuidador dispensado a esse paciente torna-se muito complexo. Assim, é comum a família obrigar-se a reajustar seus papéis para facilitar o enfrentamento da situação especifica no âmbito doméstico (16) dividindo tarefas, algo que não encontrado no perfil dos cuidadores do nosso estudo, apontando que $53,33 \%$ dos cuidadores excutam os cuidados sozinhos, favorecendo ainda mais a sobrecarga física e mental dessa população.

Pinto et al. (20), realizaram um estudo abordando o sexo dos cuidadores e avaliaram o estado emocional, a saúde mental, as mialgias (doença física) e a capacidade física e concluíram que a saúde mental, a estrutura emocional e a doença física são muito frequentemente afeta- das, de modo negativo, em mulheres, em quanto nos homens cuidadores apenas a capacidade física encontrou-se significativamente prejudicada, dados que vem de encontro com nossa pesquisa, a qual apontou um número maior de cuidador do sexo feminino $(86,66 \%)$ e, desta forma, mais vulneráveis a sobrecarga.

Estes dados também estão de acordo com um estudo multicêntrico, no qual a qualidade de vida de cuidadores principais de idosos com alterações neurológicas se relacionou com à duração da evolução da doença, tendo-se verificado que as cuidadoras mulheres têm pior qualidade de vida e maior índice de depressão do que cuidadores homens. (20)

Pinto et al. (20), concluíram que cuidadores mulheres, com idade maior que 56 anos, casadas, com filhos, níveis socioeconômico baixo, escolaridade baixa e que cuidam de idosos em estagio avançado de alguma patologia experimentaram maior sobrecarga e, consequentemente, apresentaram piora significativa da sua qualidade de vida , tal perfil de cuidador foi encontrado em nossa pesquisa e exposto na tabela 1 .

O estudo de Bocchi (21) mostra os fatores limitantes da vida social do cuidador de um indivíduo que teve Acidente Vascular Encefálico (AVE). A sobrecarga de atividades com o paciente e o acúmulo de atividades de trabalho com as tarefas de casa faz com que os cuidadores sintam-se ocupados e com limitações do tempo livre, como conseqüência direta por estarem assumindo o cuidado do ente. Tendo em vista que em nossa pesquisa os voluntários responderam que destinam 21,93 $\mathrm{h}$ /dia de cuidados, é possível que favoreça a uma sobrecarga negativa e impactante na qualidade de vida desses cuidadores.

Os distúrbios comportamentais do portador de AVE, como a apatia, a indiferença, a falta de motivação, a irritabilidade e a própria dependência do paciente levam os cuidadores a se recusarem em sair sozinhos ou a impor limites no período de passeio gerando insatisfação antecipada ao evento, promovendo o confinamento e as alterações comportamentais, afetando mudanças nos relacionamentos familiares e no círculo de amizade. Nas relações familiares podem ocorrer o aumento dos conflitos ou maior aproximação entre membros. Os laços com parentes mais próximos geralmen- 
te são preservados e reforçados após o AVC, mas mesmo assim os conjugues se queixam das limitações em encontrarem outros parentes ou amigos, bem como vivenciam a redução de visitas de amigos com o passar do tempo. Em nossa pesquisa, $73,33 \%$ dos pacientes que recebem cuidados são sequelados de AVE, indicando que o que foi descrito pelo autor está sujeito a ocorrer com essa população avaliada em nossa pesquisa. $(20,21)$

Segundo nossa pesquisa realizada com 15 cuidadores informais, que convivem com familiares que possui alguma dessas patologias relacionadas acima, verificou-se a a sobrecarga destes cuidadores através da escala de Burden Interview, sendo o questionário composto pelos seguintes domínios: Tensão Geral 23,66\%, Isolamento 8,33\%, Decepção 12,53\%, Envolvimento 5,26\%, Ambiente $6,93 \%$, obtendo um score total de sobrecarga de 56,73\%. Analisando, destaca-se Tensão Geral com a maior média de sobrecarga, onde este quesito está relacionado à responsabilidade para o bem estar do parente, além do cansaço, esgotamento, prejuízo para a saúde do cuidador e falta de tempo para si mesmo.

Para Cattani et al. (22) o esgotamento físico e emocional relatado pelas cuidadoras, está associado a múltiplos fatores. Além das tarefas dos cuidados, sofrem pressão cotidiana decorrentes do próprio estado de saúde do doente, que gera a dependência física e emocional; da falta de ajuda de outros familiares e das dificuldades financeiras para a manutenção da própria família.

O cuidador familiar de idosos incapacitados precisa ser alvo de orientação de como proceder nas situações mais difíceis. Receber em casa periódicas visitas de profissionais, médico, pessoal de enfermagem, de fisioterapia e outras modalidades de supervisão e capacitação. É preciso, também, chamar a atenção dos profissionais de saúde que indicam cuidados em casa a idosos dependentes, que sejam consideradas as mudanças sociais e econômicas que estão transformando as estruturas familiares nas cidades brasileiras e como estas podem afetar a posição transformando as estrutura familiares nas cidades brasileiras e como estas podem afetar a posição e o papel tradicional do cuidador de idosos fragilizados e dependentes. (23)

\section{CONCLUSÃO}

Neste estudo pudemos constatar que o nível de sobrecarga dos cuidadores de pacientes acamados encontra-se elevados em todos os domínios da escala de Burden Interview. Esses resultados demonstram que quando o paciente se depara com o ambiente familiar, as respostam tendem a ser mais positivas, pois se sente mais confortável em sem domínio e com os entes queridos. Entretanto, o que representa melhoras ao paciente, pode ser um sobrecarga ao cuidador, que muitas vezes não é acompanhado pelas equipes que prestam assistência ao doente. Desta forma, apesar de contribuirmos para uma melhor qualidade de vida do internado, pode-se criar uma situação desfavorável ao cuidador. Por esse motivo, acreditamos que nosso estudo pode ser uma ferramenta válida para corroborar com a importância do atendimento multidisciplinar e programas de saúde específicos ao cuidador informal e não apenas ao ser cuidado.

\section{REFERENCIAS}

1. IBGE. Instituto Brasileiro de Geografia e Estatística. Políticas do Idoso. (Estudos \& Pesquisas - Informações demográfica e socioeconômica.) Disponível em: http:// www.ibge.gov.br.

2. Silva MG, Boemer MR. Vivendo o Envelhecer: Uma perspectiva fenomenológica. Rev. Latino-am Enfernagem17(3), maio/junho 2009.

3. Maia FOM, Duarte YAO, Ledrão ML. Análise dos óbitos em idosos no Estudo SABE. Rev Esc Enferm USP. 2006; 40(4): 540-7.

4. Sampaio LFR, Boaretto MC. Envelhecimento e saúde da pessoa idosa. Ministério da Saúde, Secretária de atenção à Saúde, Departamento de Atenção Básica. 2006; $1^{\circ}$ ed., Brasília.

5. Karsch U. Idosos dependentes: famílias e cuidadores. Cad Saúde Pública. 2003; 19(3):861-866

6. Martins T, Ribeiro JP, Garrett C. Estudo de validação dos Questionário de avaliação da sobrecarga para cuidadores informais. Psicologia, Saúde e Doenças. 2003; 4(1):131-148.

7. Mazo GZ, Cardoso FL, Aguiar DL. Programa de hidroginástica para idosos: motivação, auto-estima e auto-imagem. Rev. Bras. De Cineantropometria \& desempenho humano. 2006; 8(6): 67-72.

8. Laham CF. Percepção de perdas e ganhos subjetivos entre cuidadores de pacientes atendidos em um programa de assistência domiciliar [dissertação]. São Paulo (SP): Faculdade de Medicina, Universidade de São Paulo; 2003. 
9. Marques A, Landim FLP, Collares PM,. Apoio social na experiência do familiar cuidador. Ciência e Saúde. Scielo Public Health, 2011; 16, 945-955, .

10. Barroso SM, Bandeira M. Sobrecarga de familiares de pacientes psiquiátricos atendidos na rede pública. Revista Psiquiatra Clin. Scielo Brasil, 2007; 34(6); 270-7, $2007 .$.

11. Garrido R. Impacto em cuidadores de idosos com demência atendidos em um serviço psicogeriátrico. Revista Saúde Pública. Scielo Brasil, 2004; 38 (6); 835-4.

12. Scazufca M. Versão brasileira da escala Burden Interview para avaliação de sobrecarga de cudadores de pessoas com doenças mentais. Revista brasileira de Psiquiatria. 2002; 24 (1): 12-17.

13. Riberto M, Miyazaki MH, Filho J, Sakamoto DH, Battistella LR. Reprodutibilidade da versão brasileira da medida da independência funcional. Acta Fisiátrica. 2001; 8(1): 45-52.

14. Riberto M, Miyazaki MH, Jucá SSH, Sakamoto H, Pinto PPN, Battistella LR. Validação da versão brasileira da medida de independência Funcional. Actab Fisiátrica. 2004; 11(2): 72-6.

15. TM. Silveira, CP, Caldas. Cuidando de idosos altamente dependentes na comunidade um estudo sobre cuidadores familiares principais. Cadernos de Saúde, Scielo Public Health. 2006; 22(8); 1629-1638

16. Caldeira APS, Ribeiro R. O enfrentamento do cuidador do idoso com Alzheimer. Arq. Ciência Saúde. 2004;11(2); 100-4

17. Fonseca MPF, Fonseca Rocha R. Desvelando o cotidiano dos cuidadores informais de idosos - Revista Brasileira. 2008; 61(6); 801.

18. Montezuma CA, Freitas MC. A família e o cuidado ao idoso dependente: estudo de caso - Revista eletrônica de enfermagem. 2009; 10(2)

19. Pereira MJSB, Filgueiras MST. A dependência no processo de envelhecimento: uma revisão sobre cuidadores informais de idosos. Revista de APS. 2008;12(1).

20. Pinto MF, Barbosa DA, Ferreti CEL, Souza LF. Qualidade de vida de cuidadores de idosos com doença de Alzheimer. Acta Paul. Scielo Brasil. 2009; 22 (5); 652-7.

21. Bocchi SCM. Vivenciando a sobrecarga ao vir-a-ser um cuidador familiar de pessoas com acidente vascular cerebral (AVC): análise do conhecimento. Revista Latino-America de Enfermagem. 2004 12(1):115

22. Cattani RB, Girardon-Perlini NMO. Cuidar do idoso doente no domicilio na voz de cuidadores familiares. Revista Eletrônica de Enfermagem.2004; 06 (02): 254-271.

23. Karsch UM. Idosos dependentes: familiares e cuidadores dependent sêniors: families and caregivers.Cad. Saúde Pública. 2003;19(3),861

Como citar este artigo:

Oliveira AR, Gongalvez GR, Loffredo MCM, Grecco LH. Avaliação da sobrecarga dos cuidadores informais através da escala de Burden Interview. Rev. Aten. Saúde. 2018;16(58):75-83. 